\title{
Long-Term Patient-Centred Follow-up in a Prospective Cohort of Patients with COVID-19
}

Elda Righi (D) - Massimo Mirandola · Fulvia Mazzaferri · Elisa Razzaboni · Amina Zaffagnini · Anna Erbogasto • Ilaria Dalla Vecchia · Nina Auerbach · Federico Ivaldi · Maria Mongardi • Pietro Minuz • Michele Milella • Sara Mehrabi · Oliviero Olivieri · Domenico Girelli · Enrico Polati · Claudio Micheletto · Evelina Tacconelli

Received: February 12, 2021 / Accepted: May 18, 2021 / Published online: June 21, 2021

(C) The Author(s) 2021

\section{ABSTRACT}

Introduction: To better define COVID-19 longterm impact we prospectively analysed patientcentred outcomes, including general health and symptom duration.

Methods: Barthel index (BI), St. George's Respiratory Questionnaire adapted to patients with

Elda Righi and Fulvia Mazzaferri contributed equally to this work.

Supplementary Information The online version contains supplementary material available at https:// doi.org/10.1007/s40121-021-00461-3.

E. Righi $(\bowtie) \cdot$ M. Mirandola · F. Mazzaferri ·

E. Razzaboni · A. Zaffagnini · A. Erbogasto .

I. D. Vecchia $\cdot$ N. Auerbach $\cdot$ F. Ivaldi $\cdot$ M. Mongardi . E. Tacconelli

Infectious Diseases Division, Diagnostics and Public Health Department, University of Verona, P.le L.A.

Scuro 10, 37134 Verona, Italy

e-mail: elda.righi@univr.it

\section{P. Minuz}

Section of General Medicine and Hypertension,

Medicine Department, University of Verona,

Verona, Italy

M. Milella

Oncology Division, Medicine Department,

University of Verona, Verona, Italy

S. Mehrabi

Radiology Division, Diagnostics and Public Health

Department, Verona University Hospital, Verona, Italy
COVID-19 (aSGRQ) and WHO Clinical Progression Scale (CPS) were measured at enrolment and at 6 weeks from the onset of symptoms. Persistence of most frequently reported symptoms was assessed at 6 weeks and, among symptomatic patients, at 12 weeks from the onset of symptoms. Predictors of impaired general health over time were identified using an ordinal multilevel multivariate model.

Results: A total of 448 patients (55\% men, median age 56 years) were enrolled. WHO-CPS showed mild, moderate and severe disease in $48 \%, 42 \%$ and $10 \%$ of patients at admission and

O. Olivieri

Internal Medicine, Medicine Department,

University of Verona, Verona, Italy

D. Girelli

Section of Internal Medicine, Medicine Department, University of Verona, Verona, Italy

E. Polati

Section of Anaesthesiology, Department of Surgery, Dentistry, Paediatrics and Gynaecology, University of Verona, Verona, Italy

C. Micheletto

Respiratory Unit, Cardiovascular and Thoracic Department, Verona University Hospital, Verona, Italy 
mild disease in all patients at follow-up, respectively. BI and aSGRQ were normal in 96\% and 93\% patients before COVID-19 but only in $47 \%$ and $16 \%$ at COVID-19 diagnosis and in $87 \%$ and $65 \%$ at 6 -week follow-up. Male gender was identified by all three assessments as a predictor of impaired general health (BI, OR 2.14, $p<0.0001$; aSGRQ, OR 0.53, $p=0.003$; WHO-CPS, OR 1.56, $p=0.01$ ). Other predictors included age, ICU admission and comorbidities (e.g. cardiovascular disease and cancer) for BI, hospital admission for aSGRQ, age and presence of comorbidities for WHO-CPS. At 6- and 12-week follow-up, $39 \%$ and $20 \%$ of patients, respectively, were still reporting symptoms. Fatigue and breathlessness were the most frequently reported symptoms.

Conclusions: Long-term follow-up facilitates the monitoring of health impairment and symptom persistence and can contribute to plan tailored interventions.

Keywords: COVID-19; Long-term follow-up; Barthel index; Saint George Respiratory Questionnaire; Symptoms persistence

\section{Key Summary Points}

Data from acute care settings measure the burden of COVID-19 as intensive care admissions or mortality, while longlasting impairment of general health is still poorly studied.

Follow-up data were prospectively collected to analyse health impairment up to 6 weeks and symptom experience up to 12 weeks from disease onset among 448 patients with COVID-19.

Barthel index, adapted Saint George's respiratory questionnaire and WHO-CPS were assessed over time through structured questionnaires.

Age, male gender, ICU admission, concomitant comorbidities and hospital admission were predictors of health impairment, with male gender identified as a risk factor by all three assessments.
At week 12, 20\% of patients were still reporting symptoms, most frequently fatigue and breathlessness.

Long-term follow-up is advised in patients with COVID-19.

\section{DIGITAL FEATURES}

This article is published with digital features, including a summary slide, to facilitate understanding of the article. To view digital features for this article go to https://doi.org/10.6084/ m9.figshare.14573679.

\section{INTRODUCTION}

The rapid spread of severe acute respiratory syndrome coronavirus 2 (SARS-CoV-2) infection led to the collection of a large amount of clinical data from acute care settings, measuring the burden of coronavirus disease 2019 (COVID-19) mainly as number of intensive care admissions and/or mortality. Long-term data from inpatients or outpatients who survived COVID-19 are increasing but still limited. People recovering from an acute respiratory syndrome may experience long-lasting impairments in physical functions [1], and previous outbreaks of coronaviruses have been associated with persistent pulmonary function impairment, fatigue and reduced quality of life even in patients with mild disease $[2,3]$.

To better define the long-term effects of COVID-19, follow-up data were prospectively collected from a cohort of patients with COVID19 , including inpatients and outpatients. The aim of this study was to analyse patient-centred outcomes, including health impairment, measured through validated questionnaires and symptom experience. 


\section{METHODS}

\section{Study Population}

Patients aged 18 years and older diagnosed with symptomatic SARS-CoV-2 infection at Verona University Hospital during the period $29 \mathrm{Fe}-$ bruary-25 April 2020 were consecutively screened for inclusion in the study. A local registry of patients with COVID-19 was designed at the beginning of the pandemic in order to collect clinical data (ClinicalTrials.gov NCT04497194). The study protocol was approved by the institutional review board (Ethics Committee for Clinical Experimentation of Verona and Rovigo) and informed consent was provided by all study participants. All procedures were in accordance with the $1964 \mathrm{Hel}$ sinki Declaration and its later amendments or comparable ethical standards. Laboratory-confirmed SARS-CoV-2 infection was diagnosed from respiratory samples by real-time multiplex reverse transcription polymerase chain reaction for simultaneous detection of three different SARS-CoV-2 targets (E, N and RdRP genes) using Allplex 2019-nCoV assay kit (Seegene, Seoul). Patients with no microbiological confirmation of SARS-CoV-2 and patients who did not complete the follow-up were not included in the study. Specifically, three hospitalised patients with negative swabs admitted with symptoms and imaging suggestive of COVID-19 and 83 patients who died during hospitalisation were excluded from the analyses. No deaths were recorded during the study follow-up.

Data collected included age, sex, ethnicity, comorbidities (e.g. hypertension, cardiovascular disease, respiratory disease, diabetes, cancer and renal impairment), body mass index $(\mathrm{BMI})>25$, chest X-ray results, hospital admission, antiviral and antibiotic treatment, and need for primary care visits. Length of hospital stay and need for ICU admission and orotracheal intubation were recorded among inpatients.

\section{Clinical Management}

According to the Centers of Disease Control and Prevention (CDC) clinical guidance for COVID-
19 management, patients were hospitalised on a case-by-case basis and admission criteria included hypoxia, radiological evidence of viral pneumonia and need for oxygen support as well as potential for deterioration $[4,5]$. Patients with mild clinical presentation who did not require hospitalisation were initially assessed at the hospital and discharged with indication of clinical monitoring and home self-isolation or directly managed at home by primary care physicians. During the study period, regional standard of care of COVID-19 consisted of hydroxychloroquine (HCQ) and/or lopinavir/ ritonavir $(\mathrm{LPV} / \mathrm{r})$. Inpatients were discharged on the basis of improvement of respiratory symptoms, irrespective of positivity of nasal swab.

\section{Patient Outcomes}

Four outcomes were assessed: (1) the Barthel index of activities of daily living, (2) an adapted version of the St. George's Respiratory Questionnaire tailored to patients with COVID-19 (aSGRQ), (3) patient's symptoms (cough, breathlessness, fatigue, anosmia, dysgeusia, myalgia) and (4) WHO Clinical Progression Scale (WHO-CPS). Patients' subjective perception of general health was also recorded on a 1 to 5 scale $(1=$ very poor, $2=$ poor, $3=$ fair, $4=$ good, 5 = very good).

Barthel index, aSGRQ and perception of general health were assessed at three time points: prior to the onset of COVID-19 ( $T_{0}$, based on retrospective patients' recollection), at the time of COVID-19 diagnosis $\left(T_{\mathrm{D}}\right)$ and after a median time of 6-8 weeks (43 days, Q1-Q3, $37-48)$ from the onset of symptoms ( $T_{1}$, followup). WHO-CPS was assessed at $T_{\mathrm{D}}$ and $T_{1}$. Patients reporting at least one of the aforementioned symptoms at $T_{1}$ (symptomatic patients) were rescheduled for a second phone interview $\left(T_{2}\right)$ to investigate persistence of symptoms after 4-6 weeks from $T_{1}$ (median time 34 days, Q1-Q3, 34-37). Maximum followup period was 12-14 weeks from the onset of symptoms. The study design is summarized in Supplementary Fig. 1.

Questionnaires were administered via inperson or phone interviews run by a nurse or a 
doctor involved in the care of patients with COVID-19. A structured case report form for data collection and training for conducting the interviews were provided to the healthcare workers involved in the study.

The Barthel index (BI) consists of a 10-item ordinal scale measuring patients' functional independence (e.g. self-care, sphincter management, transfers and locomotion) [6]. Total possible scores ranged from 0 to 100 , with lower scores indicating increased disability (less than 20, totally dependent; 20-39, very dependent; $40-59$, partially dependent; 60-79, minimally dependent; 80-100, independent). The adapted Saint George Respiratory Questionnaire (aSGRQ) was based on SGRQ and its short version (SGRQ-C) that measure health impairment in respiratory diseases, including infections such as tuberculosis and aspergillosis [7-10]. The aSGRQ excluded symptom characteristics of asthma (e.g. wheezing) and questions related to social activities since the study was run when patients were self-isolated at home. The aSGRQ included a symptom component (questions 1-3) assessing patients' frequency of respiratory symptoms (e.g. cough, sputum production, breathlessness) and an activity component (questions 4-6), addressing disturbances to daily activities (including perception of chest condition as a problem, occurrence of breathlessness while talking, getting washed or dressed, or walking around the house; sleep disturbances caused by chest issues, and getting easily exhausted). Higher SGRQ scores indicated increased health impairment. In order to adapt SGRQ for patients with COVID-19, we adjusted the weighting system, excluding from the total the weights of the unused items. A total score (1410.9) and two-component scores (symptoms, 446.5 and activity, 964.4) were calculated according to a predefined algorithm [11]. Individual scores were calculated using the algorithm suggested, dividing the sum of all items by the total weights of each scale and then multiplying by 100 .

The WHO-CPS indicated mild (ambulatoryno symptoms, 1; no limitation of activities, 2; limitation of activities, 3), moderate (hospitalized-no oxygen therapy, 4; oxygen by mask or nasal prongs, 5) and severe disease (oxygen by non-invasive ventilation or high flow, 6; intubation and mechanical ventilation, 7; mechanical ventilation with $\mathrm{SpO} 2 / \mathrm{FiO} 2<200$ or vasopressors, 9; mechanical ventilation with $\mathrm{pO}_{2} / \mathrm{FiO}_{2}<150$ and vasopressors, dialysis or extracorporeal membrane oxygenation [ECMO], 9) [12].

\section{Statistical Analyses}

For continuous variables, mean and standard deviation or median and IQR were calculated. For nominal variables, count and percentages were used. Wilcoxon-Mann-Whitney test was used for comparing independent groups. The association between categorical variables was assessed using Fisher's test. A multivariate logistic regression model was used to investigate the association between hospitalisation and demographic variables and comorbidities. Ordinal multilevel multivariate logistic regression with a random component was used to identify factors associated with the ordinal distribution of outcome measures such as Barthel Index, aSGRQ score and WHO-CPS over time. Proportional odds (PO) ordinal multilevel logistic regression is a well-characterised method of analysing data where a dependent variable $(Y)$ that takes ordered integer values is modelled as a function of a vector $X$ of covariates. Ordinal logistic regression fits ordered logit models of ordinal variable and provides an estimate of a set of predictors association. In order to account for the longitudinal nature of the study, the variable time was added to the model and a random effect was added to account for repeated measures at the subject level. Age, gender, comorbidities, hospitalisation and ICU admission were used as predictors of the outcome measures.

Predictors associated with the outcome variable with a probability $<0.05$ were considered significant. To investigate symptoms duration, the product-limit method (Kaplan and Meier) with $95 \%$ CI was used. Comparison of symptoms between inpatients and outpatients was performed using the log-rank test analysing each symptom separately. Analyses were carried 
out using Stata ${ }^{\circledR}$ Version 16.1 (College Station, TX: StataCorp LP).

\section{RESULTS}

Between 29 February and 25 April 2020, 531 out of 602 patients with COVID-19 screened for inclusion agreed to be enrolled in the study; 83 inpatients who died during hospitalisation were excluded from the analysis. A total of 448 patients (median age 56 years, Q1-Q3, 45-66) were available for the follow-up. Table 1 summarises the population demographic characteristics. Most patients were men (55\%) and of Italian origin (96\%). A total of $235(52 \%)$ patients required hospitalisation. Median length of stay was 10 days (Q1-Q3, 6-17). Fortyseven $(20 \%)$ patients were admitted to the ICU; of these, $87 \%$ required orotracheal intubation. Chest X-rays documented viral pneumonia in $79 \%$ of patients $(90 \%$ inpatients and $50 \%$ outpatients). Compared with those managed as outpatients, inpatients were generally older and more likely to be male with comorbidities. Specifically, a multivariate logistic regression analysis showed that age (OR 1.05, 95\% CI 1.03-1.07, $p<0.001$ ), male gender (OR 3.77, 95\% CI 2.29-6.21, $p<0.001$ ), hypertension (OR $3.04,95 \%$ CI $1.61-5.73, p=0.001)$, cardiovascular disease (OR 6.90, CI 1.98-24.09, $p=$ 0.002), respiratory disease (OR 4.45, 95\% CI $1.34-14.78, p=0.015)$ and cancer (OR 6.35, 95\% CI $1.30-30.93, p=0.02$ ) were independent risk factors for hospitalisation due to COVID-19. Antiviral therapy was provided in 93\% inpatients (HCQ and/or LPV/r) and 9\% outpatients (HCQ) $(p<0.001)$. The need for medical check among inpatients after hospital discharge and those in the outpatient group was similar (7\% and $6 \%$, respectively), while hospitalisation or, for inpatients, rehospitalisation after discharge was more commonly required for inpatients (11\% vs. $3 \%, p<0.002)$.

Patients' perception of general health (very poor $=1$; poor $=2$; fair $=3$; good $=4$; very good $=$ 5), BI score $(0-20=$ total dependency; $21-60=$ severe dependency; 61-90 = moderate dependency; $90-99=$ slight dependency; $100=$ total independency) and aSGRQ ( 0 = no limitation;
$1-10=$ slight limitation; $11-30=$ moderate limitation; greater than $30=$ severe limitation) results at different time points are summarised in Fig. 1 and Table 2. At $T_{1}$, the proportion of patients with optimal general health perception, BI and aSGRQ scores was still lower compared with values documented pre-COVID-19 (Table 1). Perception of general health was very poor or poor in $33 \%$ of patients at $T_{\mathrm{D}}$ and only in $8 \%$ of patients at $T_{1}$. BI was 100 in $47 \%$ of patients at $T_{\mathrm{D}}$ and increased to $87 \%$ at $T_{1}$. Among patients with COVID-19 at $T_{\mathrm{D}}, \mathrm{BI}$ indicated moderate or slight dependency in $25 \%$ and $20 \%$, respectively. aSGRQ was reported normal in $16 \%$ of patients at $T_{\mathrm{D}}$ and $65 \%$ at $T_{1}$,

Table 1 Characteristics of patients with COVID-19 $(n=448)$

\begin{tabular}{ll}
\hline Characteristic & Overall $(\boldsymbol{n}=\mathbf{4 4 8})$ \\
\hline Age, years & $56.0(45.0-66.0)$ \\
Sex & $202(45.1)$ \\
Female & $246(54.9)$ \\
Male & \\
Ethnicity & $440(98.2)$ \\
White & $8(1.8)$ \\
Non-white & $244(54.5)$ \\
No comorbidities & $123(27.5)$ \\
Hypertension & $65(14.5)$ \\
BMI $>25$ & $55(12.2)$ \\
Cardiovascular disease & $30(6.7)$ \\
Diabetes & $33(7.3)$ \\
Respiratory disease & $19(4.2)$ \\
Cancer & $11(2.4)$ \\
Renal impairment & $52(11.6)$ \\
Other comorbidities ${ }^{\mathrm{a}}$ & \\
\hline
\end{tabular}

${ }^{a}$ Other comorbidities included thyroid disorders $(n=28)$, rheumatological disease $(n=8)$, liver disease $(n=6)$, neurological disease $(n=6)$, solid organ transplantation $(n=2)$, human immunodeficiency virus infection $(n=2)$ 

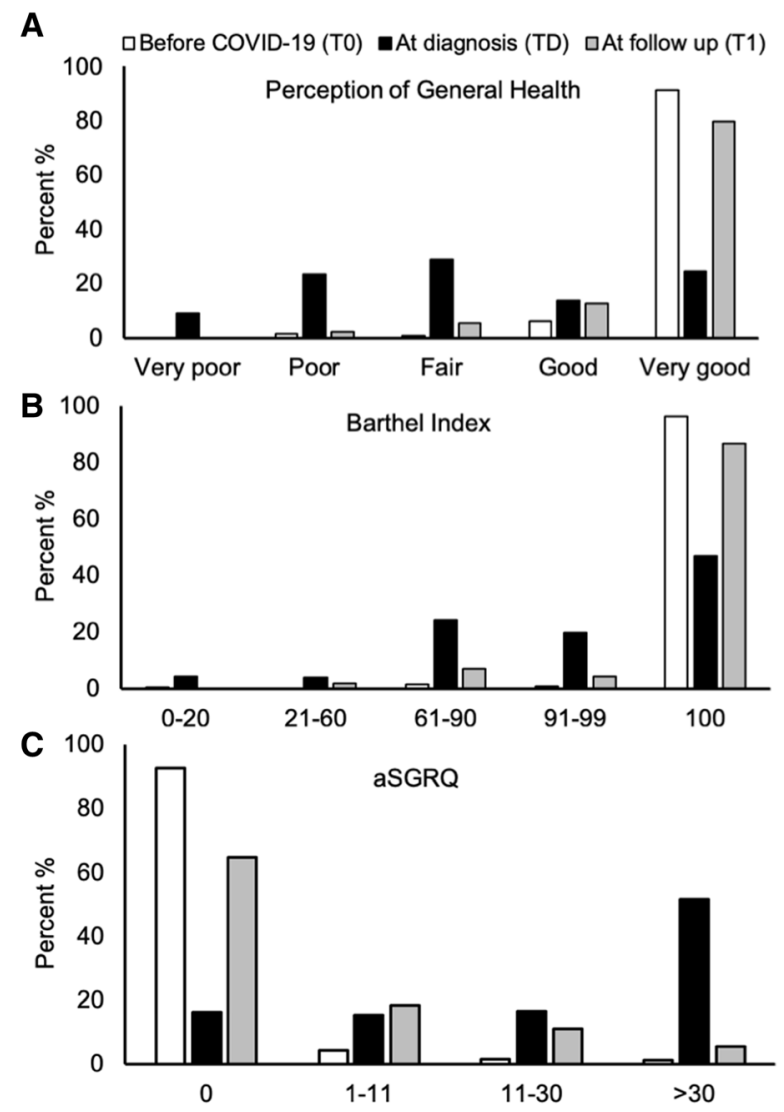

Fig. 1 General health perception, Barthel index and adapted Saint George's Respiratory Questionnaire (aSGRQ) before COVID-19 (T0), at diagnosis (TD) and at 6-week follow-up (T1)

while severe limitation was shown in over half $(52 \%)$ at $T_{\mathrm{D}}$ and only $6 \%$ at $T_{1}$ (Fig. 1 ).

Predictors significantly associated with BI or aSGRQ scores are summarised in Table 3. There was considerable improvement in BI (OR 11.2, $p<0.0001$ ) and aSGRQ (OR 0.016, $p<0.0001$ ) between $T_{\mathrm{D}}$ and $T_{1}$.

Predictors of decreased $\mathrm{BI}$ included age (OR $0.98 ; p=0.001)$, male gender (OR 2.14; $p<$ 0.0001 ), ICU admission (OR 0.19; $p<0.0001$ ) and comorbidities such as cardiovascular disease (OR 0.53; $p=0.036$ ) or malignancy (OR $0.39 ; p=0.030)$. Predictors of worse aSGRQ were male gender (OR 0.53; $p=0.003)$ and hospital admission (OR 28.0; $p<0.0001$ ).

The WHO-CPS showed significant improvement between $T_{\mathrm{D}}$ and $T_{1}$ (OR 0.014; $p<0.0001)$. Mild (2-3), moderate (4-5) and severe $(>6)$ disease were reported in $48 \%, 42 \%$ and $10 \%$ of patients at $T_{\mathrm{D}}$ (Supplementary Table 2). At $T_{1}$, all patients had mild disease; $58 \%, 29 \%$ and $13 \%$ scored 1 (asymptomatic), 2 (symptomatic independent) or 3 (symptomatic with need for assistance), respectively. As reported in Table 4, increased age (OR 1.0; $p<$ $0.0001)$ and male gender (OR 1.6; $p=0.014$ ) were significantly associated with higher WHOCPS, while absence of comorbidities was associated with lower WHO-CPS (OR 0.3; $p<$ 0.0001).

At $T_{1}, 175 / 448$ (39\%) patients were still symptomatic. Fatigue (26\%), cough (12\%), breathlessness $(11 \%)$, anosmia (8\%), dysgeusia (5\%) and myalgia (5\%) were the most commonly reported symptoms. Among 175 symptomatic patients at $T_{1}, 27$ were lost at follow-up and 148 were contacted for a second phone interview (Supplementary Fig. 1). At $T_{2}, 83 / 421$ (20\%) still reported at least one symptom, most frequently fatigue (9\%) and breathlessness (9\%), while other symptoms were less frequently reported $(<5 \%)$.

Figure 2 summarises the Kaplan-Meier curves used to illustrate overall symptom duration over time. Median duration of cough and breathlessness was 10 days (Q1-Q3, 7-17 and $5-20$, respectively), 13 days for dysgeusia and myalgia (Q1-Q3, 7-25 and 7-35, respectively) and 15 days for fatigue and anosmia (Q1-Q3, 7-51 and 7-34, respectively). Supplementary Fig. 2 compares symptom duration among inpatients and outpatients. Significantly longer duration of fatigue and dysgeusia was reported among inpatients compared to outpatients (22 vs. 14 days and 33 vs. 10 days respectively, rank $p<0.0001)$.

\section{DISCUSSION}

Long-term analyses including patients with COVID-19 after hospital discharge or patients not requiring hospital admission remain limited, although scientific communities currently encourage data dissemination [13-20]. To our knowledge, to date this is one of the largest studies collecting longitudinal long-term data from both inpatients and outpatients with 
COVID-19. As previously reported, inpatients were more likely to be male with increased age and comorbidities compared with outpatients $[21,22]$. Inpatients usually had longer duration of symptoms, especially fatigue. We also observed high rates of oral antibiotic use, especially among outpatients, highlighting the need for antimicrobial stewardship programs in patients with viral respiratory diseases.

Few articles have assessed the persistence of symptoms and perception of general health after recovery from COVID-19, highlighting a persistence of respiratory symptoms from 4 weeks and up to 3 months after acute disease [13-20]. A recent, large Chinese study including data only from hospitalised patients evidenced the persistence of fatigue up to 6 months after the onset of symptoms [19].

Previous studies have shown that fatigue represents a common symptom in ARDS survivors that can last for several months [1-3]. In our study, $26 \%$ and $25 \%$ of patients interviewed at $T_{2}$ still reported fatigue and breathlessness. Consistent with this, we observed that, while cough generally resolved between 6 and 12 weeks in the majority of patients, breathlessness and fatigue along with ageusia and dysgeusia persisted. We performed a thorough assessment of respiratory symptoms during various activities using aSGRQ, showing highest impairment among men and patients who were previously hospitalised for COVID-19. Two

Table 2 General health perception, Barthel index and adapted Saint George's Respiratory Questionnaire (aSGRQ) scores at different time points

\begin{tabular}{llll}
\hline Scale and descriptor & Before COVID-19 $\left(\boldsymbol{T}_{\mathbf{0}}\right)$ & At diagnosis $\left(\boldsymbol{T}_{\mathbf{D}}\right)$ & At follow-up $\left(\boldsymbol{T}_{\mathbf{1}}\right)$ \\
\hline General health perception (\%) & & $41(9.15)$ & 0 \\
Very poor (1) & $6(1.34)$ & $106(23.66)$ & $10(2.23)$ \\
Poor (2) & $4(0.89)$ & $130(29.02)$ & $24(5.36)$ \\
Fair (3) & $28(6.25)$ & $61(13.62)$ & $57(12.72)$ \\
Good (4) & $410(91.52)$ & $110(24.55)$ & $357(79.69)$ \\
Very good (5) & & & \\
Barthel Index (BI) (\%) & $3(0.61)$ & $20(4.46)$ & $1(0.22)$ \\
Total dependency (0-20) & $1(0.20)$ & $18(4.02)$ & $8(1.79)$ \\
Severe dependency (21-60) & $8(1.64)$ & $110(24.55)$ & $32(7.16)$ \\
Moderate dependency (61-90) & $5(1.02)$ & $90(20.09)$ & $19(4.25)$ \\
Slight dependency (91-99) & $431(96.21)$ & $210(46.88)$ & $387(86.58)$ \\
Independency (100) & & & \\
Adapted SGRQ (\%) & $415(92.63)$ & $73(16.29)$ & $290(64.73)$ \\
No limitation (0) & $20(4.46)$ & $69(15.40)$ & $83(18.53)$ \\
Slight limitation (1-10) & $7(1.56)$ & $75(16.74)$ & $50(11.16)$ \\
Moderate limitation (11-30) & $6(1.34)$ & $231(51.56)$ & $25(5.58)$ \\
Severe (> 30) & &
\end{tabular}

$T_{0}=$ baseline (prior to COVID-19); $T_{\mathrm{D}}=$ COVID-19 diagnosis; $T_{1}=6$-week follow-up

Data reported from 448 patients (results available from 447 patients for BI at $T_{1}$ )

Barthel index: lower scores indicating increased disability; aSGRQ: higher scores correspond to increased health impairment (increased symptom severity and activity limitations) 
Table 3 Predictors of decreased Barthel index (BI) and increased adapted Saint George Respiratory Questionnaire (aSGRQ) among patients with COVID-19 using an ordinal multilevel multivariate model

\begin{tabular}{|c|c|c|c|c|c|c|}
\hline & \multicolumn{3}{|c|}{ Bivariate analysis } & \multicolumn{3}{|c|}{ Multivariate analysis } \\
\hline & $\overline{\text { OR }}$ & $95 \% \mathrm{CI}$ & $p$ value & $\overline{\mathrm{OR}}$ & $95 \% \mathrm{CI}$ & $p$ value \\
\hline \multicolumn{7}{|l|}{ Barthel index } \\
\hline Age & 0.982 & $0.973-0.991$ & $<0.0001$ & 0.979 & $0.966-0.991$ & 0.001 \\
\hline Male gender & 1.535 & $1.168-2.017$ & 0.002 & 2.142 & $1.448-3.170$ & $<0.0001$ \\
\hline Hospitalisation & 0.970 & $0.739-1.273$ & 0.824 & & & \\
\hline ICU admission & 0.407 & $0.260-0.636$ & $<0.0001$ & 0.188 & $0.099-0.357$ & $<0.0001$ \\
\hline No comorbidities & 0.797 & $0.606-1.048$ & 0.104 & & & \\
\hline Hypertension & 0.829 & $0.611-1.126$ & 0.230 & & & \\
\hline $\mathrm{CV}$ disease & 0.579 & $0.387-0.867$ & 0.008 & 0.533 & $0.296-0.960$ & 0.036 \\
\hline Respiratory disease & 0.741 & $0.446-1.232$ & 0.248 & & & \\
\hline Diabetes & 0.906 & $0.523-1.570$ & 0.724 & & & \\
\hline Cancer & 0.407 & $0.221-0.748$ & 0.004 & 0.385 & $0.163-0.911$ & 0.030 \\
\hline Renal impairment & 0.583 & $0.248-1.368$ & 0.215 & & & \\
\hline $\mathrm{BMI}>25$ & 0.742 & $0.505-1.091$ & 0.129 & & & \\
\hline \multicolumn{7}{|l|}{ aSGRQ } \\
\hline Age & 1.036 & $1.027-1.046$ & $<0.0001$ & 1.014 & $0.998-1.030$ & 0.082 \\
\hline Male gender & 1.312 & $1.013-1.699$ & 0.040 & 0.532 & $0.351-0.805$ & 0.003 \\
\hline Hospitalisation & 6.909 & $5.160-9.251$ & $<0.0001$ & 27.960 & $13.540-57.741$ & $<0.0001$ \\
\hline ICU admission & 2.838 & $1.871-4.305$ & $<0.0001$ & 1.559 & $0.833-2.918$ & 0.165 \\
\hline No comorbidities & 2.946 & $2.261-3.837$ & $<0.0001$ & 1.076 & $.589-1.966$ & 0.811 \\
\hline Hypertension & 2.378 & $1.788-3.163$ & $<0.0001$ & 1.363 & $0.760-2.445$ & 0.299 \\
\hline $\mathrm{CV}$ disease & 2.677 & $1.824-3.930$ & $<0.0001$ & 1.372 & $0.742-2.537$ & 0.314 \\
\hline Respiratory disease & 2.297 & $1.423-3.707$ & 0.001 & 1.379 & $0.666-2.860$ & 0.387 \\
\hline Diabetes & 1.716 & $1.038-2.838$ & 0.035 & 0.735 & $0.341-1.582$ & 0.431 \\
\hline Cancer & 2.525 & $1.353-4.709$ & 0.004 & 1.664 & $0.662-4.185$ & 0.279 \\
\hline Renal impairment & 1.709 & $0.788-3.711$ & 0.175 & & & \\
\hline $\mathrm{BMI}>25$ & 2.740 & $1.909-3.932$ & $<0.0001$ & 1.105 & $0.637-1.919$ & 0.722 \\
\hline
\end{tabular}

$T_{1}=6$-week follow-up

Changes in Barthel Index were significantly different between $T_{\mathrm{D}}$ (COVID-19 diagnosis) and $T_{1}$ (OR 11.195, 95\% CI 7.329-17.102, $p<0.0001$ )

Changes in aSGRQ were significantly different between $T_{\mathrm{D}}$ (COVID-19 diagnosis) and $T_{1}$ (OR 0.016, 95\% CI $0.008-0.033, p<0.0001)$ 
Table 4 Predictors of WHO-CPS among patients with COVID-19 using an ordinal multilevel multivariate model

\begin{tabular}{|c|c|c|c|c|c|c|}
\hline \multirow[t]{2}{*}{ Factor } & \multicolumn{3}{|c|}{ Bivariate } & \multicolumn{3}{|c|}{ Multivariate } \\
\hline & $\overline{\mathrm{OR}}$ & 95\% CI & $p$ value & $\overline{\text { OR }}$ & 95\% CI & $p$ value \\
\hline Age & 1.021 & $1.014-1.030$ & $<0.0001$ & 1.026 & $1.012-1.039$ & $<0.0001$ \\
\hline Male gender & 1.506 & $1.187-1.910$ & 0.001 & 1.556 & $1.094-2.213$ & 0.014 \\
\hline ICU admission & 2.665 & $1.793-3.961$ & $<0.0001$ & & & \\
\hline No comorbidities & 2.393 & $1.874-3.056$ & $<0.0001$ & 0.284 & $0.171-0.471$ & $<0.0001$ \\
\hline $\mathrm{CV}$ disease & 2.013 & $1.394-2.907$ & $<0.0001$ & 1.045 & $0.605-1.804$ & 0.874 \\
\hline Hypertension & 1.842 & $1.406-2.414$ & $<0.0001$ & 0.614 & $0.370-1.022$ & 0.061 \\
\hline Renal impairment & 1.729 & $0.829-3.606$ & 0.145 & & & \\
\hline Respiratory disease & 1.576 & $0.985-2.523$ & 0.058 & & & \\
\hline Diabetes & 1.649 & $1.035-2.625$ & 0.035 & 0.886 & $0.452-1.735$ & 0.723 \\
\hline Cancer & 1.649 & $0.941-2.889$ & 0.081 & & & \\
\hline $\mathrm{BMI}>25$ & 2.019 & $1.419-2.874$ & $<0.0001$ & 1.437 & $0.878-2.350$ & 0.149 \\
\hline
\end{tabular}

$T_{1}=$ 6-week follow-up

Changes in WHO-CPS were significantly different between $T_{\mathrm{D}}$ (COVID-19 diagnosis) and $T_{1}$ (OR 0.014, 95\% CI $0.008-0.022, p<0.0001)$
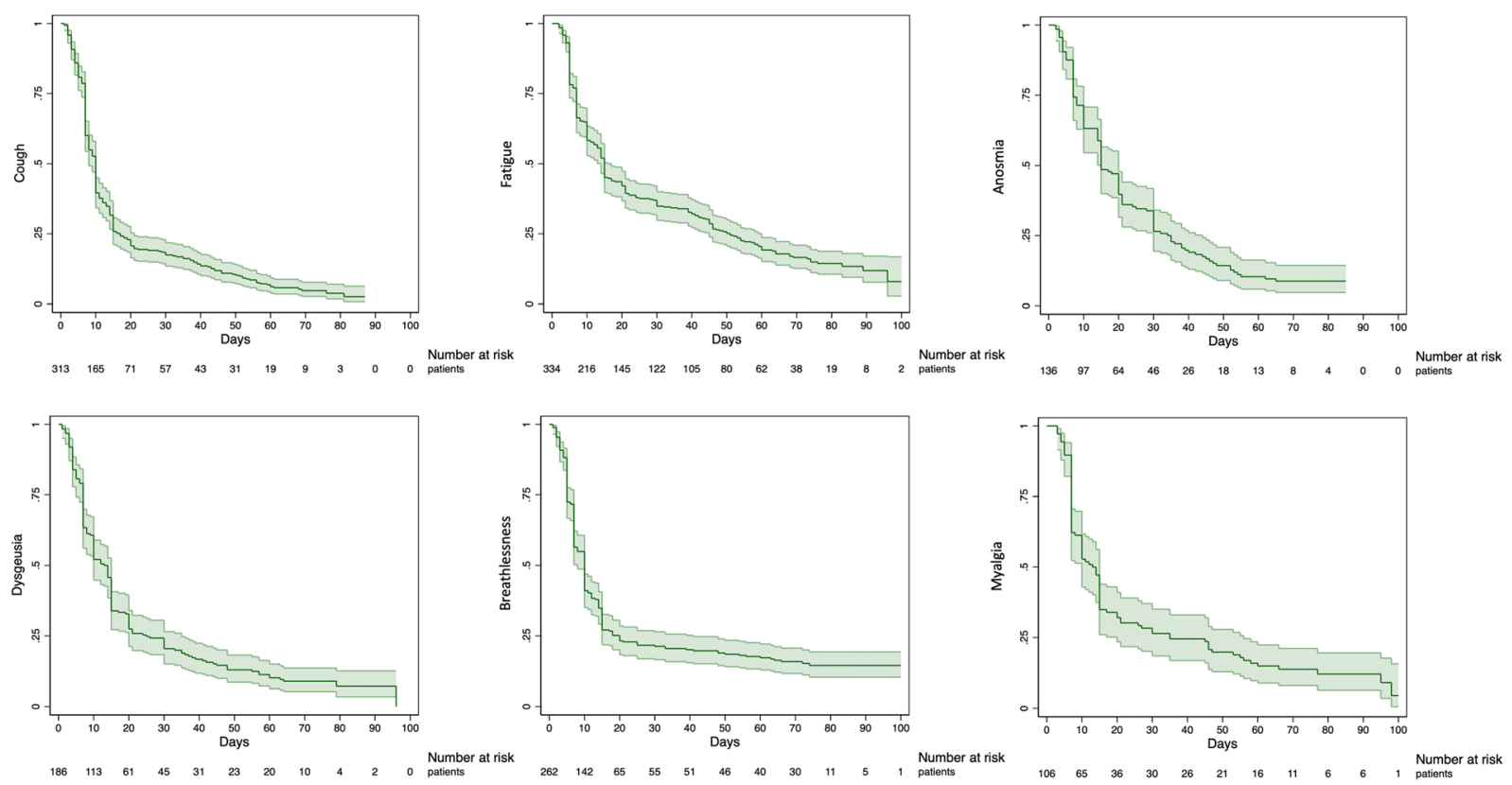

Fig. 2 Kaplan-Maier curves with 95\% confidence interval reporting duration of symptoms among patients with COVID-19

studies encompassing 143 and 120 patients, discharged from hospital and evaluated at 2 and 3 months from symptom onset, reported the persistence of fatigue in $55 \%$ and $53 \%$ of subjects, respectively $[14,20]$, and one report 
identified hospital admission as risk factor for symptom persistence at day 60 [17].

The Barthel index represents a widely used test to assess functional status in various patient populations and has shown reliability also if administered telephonically [23]. Its use is promising in patients with COVID-19 and showed profound impairment at diagnosis independency (dropping from 95\% before COVID-19 to $47 \%$ ) and persistence of various degree of dependency in $13 \%$ of patients. Factors that are common in severe forms of COVID-19 such as age, male gender, ICU admission and presence of comorbidities (e.g. cardiovascular disease and concomitant malignancy) were independent factors for having a decreased Barthel index. Owing to its use in the follow-up of acute infectious respiratory disease, also aSGRQ represents another promising tool to incorporate in the evaluation of patients recovering from COVID-19. SGRQ has been previously used to assess health-related quality of life in patients recovering from ARDS related to influenza A H1N1, showing substantial improvement only between 3 and 6 months following acute disease [24]. In our study, we observed improved, yet still worse compared to baseline, aSGRQ at $T_{1}$ in inpatients. Male gender and hospital admission were significantly associated with decreased quality of life measured through aSGRQ.

Disease severity was assessed using the WHO-CPS at $T_{\mathrm{D}}$ but was also used to evaluate symptoms persistence and dependency at $T_{1}$. In our population, the majority of hospitalised patients showed moderate disease, while patients who were not hospitalised were mainly independent and did not require assistance. Absence of comorbidities was a predictor of lower WHO-CPS, while age and male gender were associated with worse scores.

Our study has several limitations. Firstly, we presented data from a single site during the initial phases of the COVID-19 pandemic in Italy; therefore the results may be less generalizable to other cohorts. Secondly, patients' conditions at $T_{0}$ were based on retrospective recollection and may not be thoroughly indicative of their general health status before COVID-19. Furthermore, SGRQ was not used in its original version but adapted to patients with COVID-19. Thirdly, we did not perform quantitative analysis but used a questionnaire to assess patients' general health. Finally, we did not perform analyses regarding the impact of treatment of COVID-19. Nevertheless, the drugs used during the study period (e.g. chloroquine and LPV/r) are not currently used and did not demonstrate substantial benefits on disease progression. Steroids, which are currently recommended for the treatment of severe cases of COVID-19, were inconsistently used in the earlier stages of the pandemic and therefore also in our study, making potential conclusions less reliable. Furthermore, outpatients did not usually receive treatment compared with inpatients.

\section{CONCLUSIONS}

Factors such as presence of comorbidities (e.g. cardiovascular disease and concomitant malignancy), increased age, male gender, hospital admission and ICU admission were identified as predictors that significantly affect quality of life among patients even after several weeks from COVID-19 recovery. We also confirmed persistence of symptoms such as breathlessness and fatigue up to 3 months from COVID-19 onset.

Our findings have relevant implications for the long-term care of patients with COVID-19 suffering from prolonged symptoms that may impair their quality of life and the return to normal daily activities. Data from structured phone interview to assess patients' general health over time may be useful when patient circulation is limited because of self-isolation and can help to allocate resources and to support the decision-making process for the management of patients following acute disease recovery.

\section{ACKNOWLEDGEMENTS}

We thank the patients and their families for providing consent and assisting with the present study. We would also like to thank the 
health professional staff involved in all COVID19 wards at Verona University Hospital.

Funding. The present study was funded by Cariverona Foundation (ENACT Fund 2020), Italy. No Rapid Service Fee was received by the journal for the publication of this article.

Authorship. All named authors meet the International Committee of Medical Journal Editors (ICMJE) criteria for authorship for this article, take responsibility for the integrity of the work as a whole, and have given their approval for this version to be published

Authorship Contributions. ER (E. Righi), FM, MM (M. Mirandola), and ET designed the study. ER, ER (E. Razzaboni), AZ, IDV, AE, NA, FI, and MM (M. Mongardi) participated in data collection and management. MM (M. Mirandola) and ER performed data analysis. ER and ET wrote the manuscript; FM, MM (M. Mirandola), PM, SM, OO, DG, EP, CM, MM (M. Milella), ET were involved in data collection and critically revised the manuscript. All authors approved the final version of the manuscript.

Disclosures. Elda Righi, Massimo Mirandola, Fulvia Mazzaferri, Elisa Razzaboni, Amina Zaffagnini, Anna Erbogasto, Ilaria Dalla Vecchia, Nina Auerbach, Federico Ivaldi, Maria Mongardi, Pietro Minuz, Michele Milella, Sara Mehrabi, Oliviero Olivieri, Domenico Girelli, Enrico Polati, Claudio Micheletto and Evelina Tacconelli have nothing to disclose.

Compliance with Ethics Guidelines. The study protocol was approved by the institutional review board (Ethics Committee for Clinical Experimentation of Verona and Rovigo) and informed consent was provided by all study participants. All procedures were in accordance with the 1964 Helsinki Declaration and its later amendments or comparable ethical standards.

Data Availability. The datasets generated during and/or analysed during the current study are available from the corresponding author on reasonable request.
Open Access. This article is licensed under a Creative Commons Attribution-NonCommercial 4.0 International License, which permits any non-commercial use, sharing, adaptation, distribution and reproduction in any medium or format, as long as you give appropriate credit to the original author(s) and the source, provide a link to the Creative Commons licence, and indicate if changes were made. The images or other third party material in this article are included in the article's Creative Commons licence, unless indicated otherwise in a credit line to the material. If material is not included in the article's Creative Commons licence and your intended use is not permitted by statutory regulation or exceeds the permitted use, you will need to obtain permission directly from the copyright holder. To view a copy of this licence, visit http://creativecommons.org/licenses/by$\mathrm{nc} / 4.0 /$.

\section{REFERENCES}

1. Ngai JC, Ko FW, Ng SS, et al. The long-term impact of severe acute respiratory syndrome on pulmonary function, exercise capacity and health status. Respirology. 2010;15:543-50.

2. Tansey CM, Louie M, Loeb M, et al. One-year outcomes and health care utilization in survivors of severe acute respiratory syndrome. Arch Intern Med. 2007;167:1312-20.

3. Neufeld KJ, Leoutsakos J-MS, Yan H, et al. Fatigue symptoms during the first year following ARDS. Chest. 2020;158:999-1007.

4. Liang W, Liang H, Ou L, et al. Development and validation of a clinical risk score to predict the occurrence of critical illness in hospitalised patients with COVID-19. JAMA Intern Med. 2020;180: 1081-9.

5. CDC. Interim clinical guidance for management of patients with confirmed coronavirus disease (COVID-19). https://www.cdc.gov/coronavirus/ 2019-ncov/hcp/clinical-guidance-managementpatients.html. Accessed on 1 Aug 2020.

6. Mahoney FI, Barthel DW. Functional evaluation: the Barthel Index. Md State Med J. 1965;14:61-5.

7. Jones PW, Quirk FH, Baveystock CM, et al. A selfcomplete measure for chronic airflow limitation - 
the St George's Respiratory Questionnaire. Am Rev Respir Dis. 1992;145:1321-7.

8. Meguro M, Barley EA, Spencer S, et al. Development and validation of an improved, COPD-specific version of the St. George Respiratory Questionnaire. Chest. 2007;132(2):456-63.

9. Al-Shair K, Atherton GTW, Kennedy D, et al. Validity and reliability of the St. George's Respiratory Questionnaire in assessing health status in patients with chronic pulmonary aspergillosis. Chest. 2013;144:623-31.

10. Saint George's University of London, Health Status Research. Saint George's Respiratory Questionnaire. http://www.healthstatus.sgul.ac.uk/sgrq/sgrqdownloads. Accessed 10 July 2020.

11. Saint George's Respiratory Questionnaire. http:// www.healthstatus.sgul.ac.uk/sgrq/sgrq-downloads. Accessed 31 Jan 2021.

12. WHO Working Group on the Clinical Characterisation and Management of COVID-19 infection. A minimal common outcome measure set for COVID19 clinical research. Lancet Infect Dis. 2020;20: e192-e197.

13. Cellai M, O'Keefe JB. Characterization of prolonged COVID-19 symptoms in an outpatient telemedicine clinic. Open Forum Infect Dis. 2020;7(10):ofaa420.

14. Garrigues E, Janvier P, Kherabi Y, et al. Post-discharge persistent symptoms and health-related quality of life after hospitalisation for COVID-19. J Infect. 2020;81(6):e4-e6.

15. Halpin SJ, McIvor J, Whyatt G, et al. Postdischarge symptoms and rehabilitation needs in survivors of COVID-19 infection: a cross-sectional evaluation. J Med Virol. 2021;93(2):1013-22.

16. Goërtz YMJ, Van Herck M, Delbressine JM, et al. Persistent symptoms 3 months after a SARS-CoV-2 infection: the post-COVID-19 syndrome? ERJ Open Res. 2020;6(4):00542-2020.

17. Carvalho-Schneider C, Laurent E, Lemaignen A, et al. Follow-up of adults with non-critical COVID19 two months after symptoms' onset. Clin Microbiol Infect. 2021;27(2):258-63.

18. Carfi' A, Barnabei R, Landi F, et al. Persistent symptoms in patients after acute COVID-19. JAMA. 2020;324:603-5.

19. Huang C, Huang L, Wang Y, et al. 6-month consequences of COVID-19 in patients discharged from hospital: a cohort study. Lancet. 2021;S0140-6736(20):32656-8.

20. Yelin D, Wirtheim E, Vetter $\mathrm{P}$, et al. Long-term consequences of COVID-19: research needs. Lancet Infect Dis. 2020;20(10):1115-7.

21. Coronavirus disease 2019 (COVID-19). Situation Report-46. World Health Organization (WHO). https://www.who.int/docs/default-source/ coronaviruse/situation-reports/20200306-sitrep-46COVID-19.pdf. Accessed 20 Aug 2020.

22. Centers for Disease Control and Prevention (CDC). Evidence used to update the list of underlying medical conditions that increase a person's risk of severe illness from COVID-19. https://www.cdc. gov/coronavirus/2019-ncov/need-extraprecautions/evidence-table.html. Accessed 1 Aug 2020.

23. Della Pietra GL, Savio K, Oddone E, et al. Validity and reliability of the Barthel index administered by telephone. Stroke. 2011;42:2077-9.

24. Hsieh MJ, Lee WC, Cho HJ, et al. Recovery of pulmonary functions, exercise capacity, and quality of life after pulmonary rehabilitation in survivors of ARDS due to severe influenza A (H1N1) pneumonitis. Influenza Other Respir Viruses. 2018;12: 643-8. 